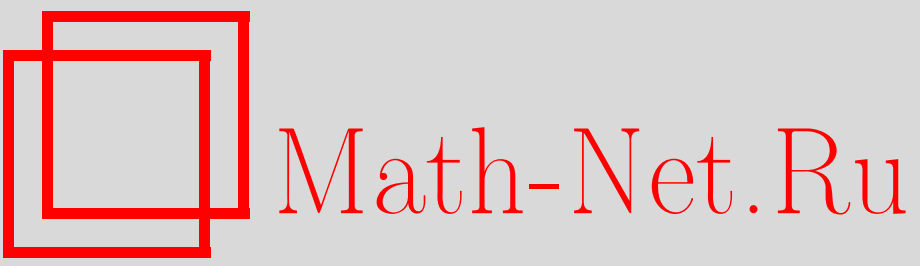

А. Г. Костюченко, К. А. Мирзоев, Критерий вполне неопределенности трехчленных рекуррентных соотношений, УМН, 1996, том 51, выпуск 6, 207-208

DOI: https://doi.org/10.4213/rm1030

Использование Общероссийского математического портала Math-Net.Ru подразумевает, что вы прочитали и согласны с пользовательским соглашением

http://www.mathnet.ru/rus/agreement

Параметры загрузки:

IP : 54.89 .56 .158

26 апреля 2023 г., 12:38:24 


\title{
КРИТЕРИЙ ВПОЛНЕ НЕОПРЕДЕЛЕННОСТИ ТРЕХЧЛЕННЫХ РЕКУРРЕНТНЫХ СООТНОШЕНИЙ
}

\author{
А. Г. Костюченко, К. А. МиРЗОев
}

1. Пусть $C^{p}(p \geqslant 1)$ - эвклидово $p$-мерное пространство вектор-столбцов со стандартным скалярным произведением $y^{*} x$, a $\ell_{p}^{2}$ - гильбертово пространство бесконечных последовательностей векторов $u=\left(u_{0}, u_{1}, \ldots\right), u_{j} \in C^{p}$, со скалярным произведением $(u, v)=\sum_{j=0}^{+\infty} v_{j}^{*} u_{j}$. Пусть, далее, $A_{j}, B_{j}(j=0,1, \ldots)$ - квадратные матрицы порядка $p$, элементы которых являются комплексными числами, причем $A_{j}$ - самосопряженная матрица, а $B_{j}^{-1}$ существует. Рассмотрим разностное выражение второго порядка

$$
(l u)_{j}=B_{j} u_{j+1}+A_{j} u_{j}+B_{j-1}^{*} u_{j-1}, \quad j=0,1, \ldots,
$$

где $u_{-1}=0, u_{0}, u_{1}, \ldots \in C^{p}$. Символом $\boldsymbol{l}$ обозначим операцию, действующую на последовательности матриц $U_{0}, U_{1}, \ldots$ размерности $p$ с элементами из $C$ так же, как и $l$. Пусть $P_{j}(z)$ и $Q_{j}(z)$ - решения матричного уравнения

$$
(\boldsymbol{l} U)_{j}=z U_{j}, \quad j=0,1, \ldots,
$$

удовлетворяющие началњным условиям $P_{1}(z)=O, P_{0}(z)=I$ и $Q_{0}(z)=O, Q_{1}(z)=B_{0}^{-1}$, соответственно, где $O$ и $I$, как обычно, нулевая и единичная матрица размерности $p$. Матриц-функции $P_{j}(z)$ и $Q_{j}(z)$ являются многочленами $j$-го и $(j-1)$-го порядка от комплексного переменного $z$ с матричными коэффициентами и назьваются матричными многочленами первого и второго рода, соответственно (см. [1, гл. VII, § 2], [2]).

Справедлива следующая

Теорема 1. Если каждое решение векторного уравнения

$$
(l u)_{j}=z u_{j}, \quad j=0,1, \ldots,
$$

для некоторого вещественного $z_{0}$ принадлежит пространству $\ell_{p}^{2}$, то для произвольного комплексного z каждое решение уравнения (2) принадлежит пространству $\ell_{p}^{2}$.

2. Рассмотрим теперь минимальный замкнутый оператор $L$, порожденный выражением $l$ и граничным условием $u_{-1}=0$ в пространстве $\ell_{p}^{2}$. Известно, что оператор $L$ симметричен, но, вообще говоря, несамосопряжен, и дефектные числа $n_{+}$и $n_{-}$этого оператора удовлетворяют неравенствам $0 \leqslant n_{+}, n_{-} \leqslant p$. Говорят, что для выражения $l$ имеет место вполне неопределенньй случай, если $n_{+}=n_{-}=p$ (см. [1], [2]). Из теоремы 1 следует, что для выражения $l$ имеет место вполне неопределенный случай тогда и только тогда, когда все решения уравнения (2) при $z=0$ принадлежат пространству $\ell_{p}^{2}$.

По аналогии с обыкновенными дифференциальными уравнениями последовательность

$$
Q_{j} P_{j}^{*}-P_{j} Q_{j}^{*}=K_{i j}, \quad i, j=0,1, \ldots,
$$

где $P_{i}=P_{i}(0)$ и $Q_{i}=Q_{i}(0)$, будем называть последовательностью Коши уравнения (1) (при $z=0)$. Эта последовательность удовлетворяет матричному уравнению (1) по $i$ при фиксированном $j(z=0)$, начальным условиям

и соотношению

$$
K_{j j}=0, \quad K_{j+1, j}=B_{j}^{-1}, \quad j=0,1, \ldots,
$$

$$
K_{i j}^{*}=-K_{j i}, \quad i, j=0,1,2 \ldots
$$

Следующая теорема является аналогом соответствующего утверждения (см. [3]) для обыкновенных симметрических дифференциальных уравнений.

Работа выполнена при финансовой поддержке Российского фонда фундаментальных исследований (грант № 96-01-00333). 
Теорема 2. Для того чтобы для выражения $l$ имел место вполне неопределенный случай, необходимо и достаточно, чтобь для любой последовательности отрезков натуральных чисел $\left[n_{k}, m_{k}\right]$ таких, что $m_{k} \leqslant n_{k+1}<m_{k+1}, k=1,2, \ldots$, выполнялось условие

$$
\sum_{k=1}^{+\infty}\left(\sum_{i=n_{k}}^{m_{k}} \sum_{j=n_{k}}^{i}\left\|K_{i j}\right\|^{2}\right)^{1 / 2}<+\infty
$$

3. Из теоремы 2 можно извлечь, что справедлива

ТеОРема 3. Пусть выполняется какое-либо из следующих условий:

а) $\sum_{k=1}^{+\infty}\left\|B_{k}^{-1}\right\|=+\infty$

б) $\sum_{k=1}^{+\infty}\left\|B_{k+1}^{-1} A_{k+1} B_{k}^{-1}\right\|=+\infty$

в) $\sum_{k=1}^{+\infty}\left\|B_{k+2}^{-1}\left(A_{k+2} B_{k+1}^{-1} A_{k+1}-B_{k+1}^{*}\right) B_{k}^{-1}\right\|=+\infty$,

Тогда для выражения $l$ не имеет места вполне неопределенный случай.

Отметим, что в случае $p=1$ условие а) теоремы 3 совпадает с известным признаком Карлемана (см., например, [1, гл. VII, $\S 1$, теорема 1.3]), а условие б) этой теоремы с признаком Денниса и Уолла (см. [3, гл. I, дополнения и задачи, 2]).

Справедлива также

Teоpema 4. Пусть $\left\|A_{k}\right\| \leqslant C(k=0,1, \ldots) u$

$$
\sum_{j=0}^{+\infty} \sum_{s=0}^{+\infty}\left\|B_{j}^{*^{-1}} B_{j+1} B_{j+2}^{*^{-1}} \cdots B_{j+2 s-1} B_{j+2 s}^{*^{-1}}\right\|<+\infty .
$$

Тогда для выражения $l$ имеет место вполне неопределенный случай.

Наметим доказательство теоремы 4 . Если $\left\|A_{k}\right\| \leqslant C$, то оператор $y_{k} \rightarrow A_{k} y_{k}(k=0,1, \ldots)$, очевидно, является ограниченным самосопряженным оператором в пространстве $\ell_{p}^{2}$. Поэтому согласно известной теореме (см., например, $[5$, гл. IV, $\S 14$, теорема 6$]$ ) дефектные числа оператора $L$ и оператора, порожденного выражением

$$
\left(l_{1} u\right)_{j}=B_{j} u_{j+1}+B_{j-1}^{*} u_{j-1}, \quad j=1,2, \ldots,
$$

совпадают. Далее следует применить теорему 2 к выражению $l_{1}$, учитывая, что здесь

$$
K_{j+2 s, j}=0, \quad K_{j+2 s+1, j}=(-1)^{s} B_{j+2 s}^{-1} B_{j+2 s-1}^{*} \cdots B_{j+1}^{*} B_{j}^{-1}, \quad s, j=0,1,2 \ldots .
$$

Теорема 4 является обобщением одной теоремп Березанского для случая $p=1$ (см., например, [1, гл. VII, §1, теорема 1.5]).

ЗАмечАниЕ. Все резултаты данной работы без изменений переносятся на случай, когда пространство $C^{p}$ заменяется на сепарабельное гильбертово пространство $H$, а матрищы $A_{j}, B_{j}$ $(j=0,1, \ldots)$ - на линейные ограниченные операторы в нем.

\section{СПИСОК ЛИТЕРАТУРЫ}

[1] Березанский Ю. М. Разложение по собственным функциям самосопряженных операторов. Киев: Наукова думка, 1965. [2] Крейн М. Г. // ДАН СССР. 1949. Т. 69. № 3. С. 125-128. [3] Мирзоев К. А. // УМН. 1991. Т. 46. №4. С. 161-162. [4] Ахиезер Н. И. Классическая проблема моментов. М.: Изд-во фииз.-мат. лит., 1961. [5] Наймарк М. А. Линейные диффференциальные операторы. М.: Наука, 1969.

Московский государственный

Принято редколлегией университет им. М.В. Ломоносова; 29.10 .1996

Московский авиационно технологический институт им. К.Э. Циолковского 